\begin{tabular}{|c|c|}
\hline Title: & $\begin{array}{l}\text { Current Control and Energy Balancing of a Square-Wave Powered 1AC-3AC Modular } \\
\text { Multilevel Converter }\end{array}$ \\
\hline Authors: & $\begin{array}{l}\text { Mathias Schnarrenberger, Felix Kammerer, Mario Gommeringer, Johannes Kolb, } \\
\text { Michael Braun }\end{array}$ \\
\hline Institute: & $\begin{array}{l}\text { Karlsruhe Institute of Technology (KIT) } \\
\text { Elektrotechnisches Institut (ETI) }\end{array}$ \\
\hline Type: & Conference Proceedings \\
\hline Published at: & $\begin{array}{l}\text { Proceedings } 2015 \text { IEEE Energy Conversion Congress and Exposition (ECCE), Montreal, } \\
\text { QC, } 2015 \\
\text { Publisher: IEEE } \\
\text { ISSN: } 2329-3721 \\
\text { Year: } 2015 \\
\text { ISBN: } 978-1-4673-7151-3 \\
\text { Pages: } 3607-3614\end{array}$ \\
\hline Hyperlinks: & DOI: $10.1109 / E C C E .2015 .7310170$ \\
\hline
\end{tabular}

(C) 2015 IEEE. Personal use of this material is permitted. Permission from IEEE must be obtained for all other uses, in any current or future media, including reprinting/republishing this material for advertising or promotional purposes, creating new collective works, for resale or redistribution to servers or lists, or reuse of any copyrighted component of this work in other works. 


\title{
Current Control and Energy Balancing of a Square-Wave Powered 1AC-3AC Modular Multilevel Converter
}

\author{
Mathias Schnarrenberger, Felix Kammerer, Mario Gommeringer, Johannes Kolb ${ }^{\ddagger}$ and Michael Braun \\ Karlsruhe Institute of Technology (KIT) \\ Elektrotechnisches Institut (ETI) - Electrical Drives and Power Electronics \\ Kaiserstr. 12, 76131 Karlsruhe, Germany \\ mathias.schnarrenberger@kit.edu, www.eti.kit.edu \\ ${ }^{\ddagger}$ Schaeffler Technologies AG \& Co. KG - SHARE at KIT
}

\begin{abstract}
This paper presents a new control method for a Modular Multilevel Converter (MMC) fed by a push-pull converter via a medium frequency (MF) transformer. Application of this topology is a universal high-precision $3 \mathrm{AC}$ voltage source for a Power Hardware-in-the-Loop Emulator with a frequency range from DC up to almost the medium frequency. Advantages are high efficiency, very low harmonic distortion and high dynamics of the output voltage which can be used e.g. for simulation of electrical machines. Due to the medium frequency input of the MMC the transformer size for the galvanic isolation is much smaller compared to a low frequency input. Additionally, the required cell capacitance for the MMC is reduced which saves cost and space. The delivered medium frequency squarewave voltage requires an alternating input current of the MMC. For the square-wave powered $1 \mathrm{AC}-3 \mathrm{AC} \mathrm{MMC}$ an energy and current control is proposed. Due to very high current dynamic requirements a dead-beat controller as subordinated controller is used to achieve a trapezoidal input current which allows zero current switching (ZCS) at the push-pull-converter. The design of the superposed energy and balancing controller is also shown. For the coupling between the superposed and subordinated control loop the arm power is analyzed and a calculation scheme is given.
\end{abstract}

Keywords-Modular Multilevel Converter (MMC), control theory, energy and balancing control, galvanic isolation, zero current switching (ZCS)

\section{INTRODUCTION}

Fig. 1 shows the investigated topology of a Modular Multilevel Converter (MMC) fed by a push-pull converter [1]. At the primary side a DC voltage source/sink supplies the $\mathrm{H}$-bridge working as a push-pull converter. The DC voltage source/sink is usually an Active Front End connected to the power grid. A galvanic isolation between the primary and secondary side is achieved by a medium frequency (MF) transformer. At the secondary side the MMC converts the power into a three phase output voltage with adjustable amplitude and frequency. Due to the multilevel topology the output voltage contains very low harmonics. For even lower harmonics in the switching frequency range a small optional low-pass filter can be installed.

MMCs are already used for galvanic isolation as shown in [2]. There, an indirect conversion from $3 \mathrm{AC}$ over $\mathrm{DC}$ to a square-wave voltage is realized using two MMCs. The squarewave voltage supplies a transformer with a diode rectifier. In [3] a direct conversion from $3 \mathrm{AC}$ to a square-wave voltage is realized using one MMC which also feeds a transformer with a diode rectifier. Both solutions allow a unidirectional power flow.

An Advantage of the investigated topology is a bidirectional power flow due to the H-bridge at the push-pullconverter. Furthermore, by use of a proper current control in the MMC, zero current switching in the push-pull-converter can be achieved. Therefore, switching losses are reduced. This is realized using a trapezoidal current in the H-Bridge which also leads to a high current utilization of the used semiconductors. Due to the medium-frequency AC input voltage of the MMC there is no singular point in the MMC operation over the output frequency range from DC up to almost the medium frequency. A singular point occurs at the MMC topology if the output frequency equals the input frequency. Additionally, the transformer size and the cell capacitance can be reduced [4]. Considering the very low harmonics of the output voltage this opens a whole area of applications for e.g. Power Hardwarein-the-Loop Emulators [5], controlling Epstein frames [6] and test equipment for measurement of sinusoidal fed electrical machines [7].

The basic principle of operation is shown in Section II. Section III presents the design of the current and energy controllers and the conversion formulas from power to current. Section IV shows simulation results. Section V summarizes the simulation and results of this paper.

\section{FUNDAMENTALS}

This Section presents the basic idea for the operation of the Modular Multilevel Converter (MMC) according to Fig. 1. The complete system includes a DC-source/sink, an H-bridge, a transformer, the MMC and an optional output filter, as shown in Fig. 1. The H-bridge of the push-pull-converter generates a medium frequency square-wave voltage at the transformer and thereby also at the input of the MMC. To achieve a high current utilization in the H-Bridge the input current should be a square wave. Due to inductors in the circuit the current is controlled trapezoidal by the MMC. With a trapezoidal current, zero current switching in the push-pull converter can be achieved. 


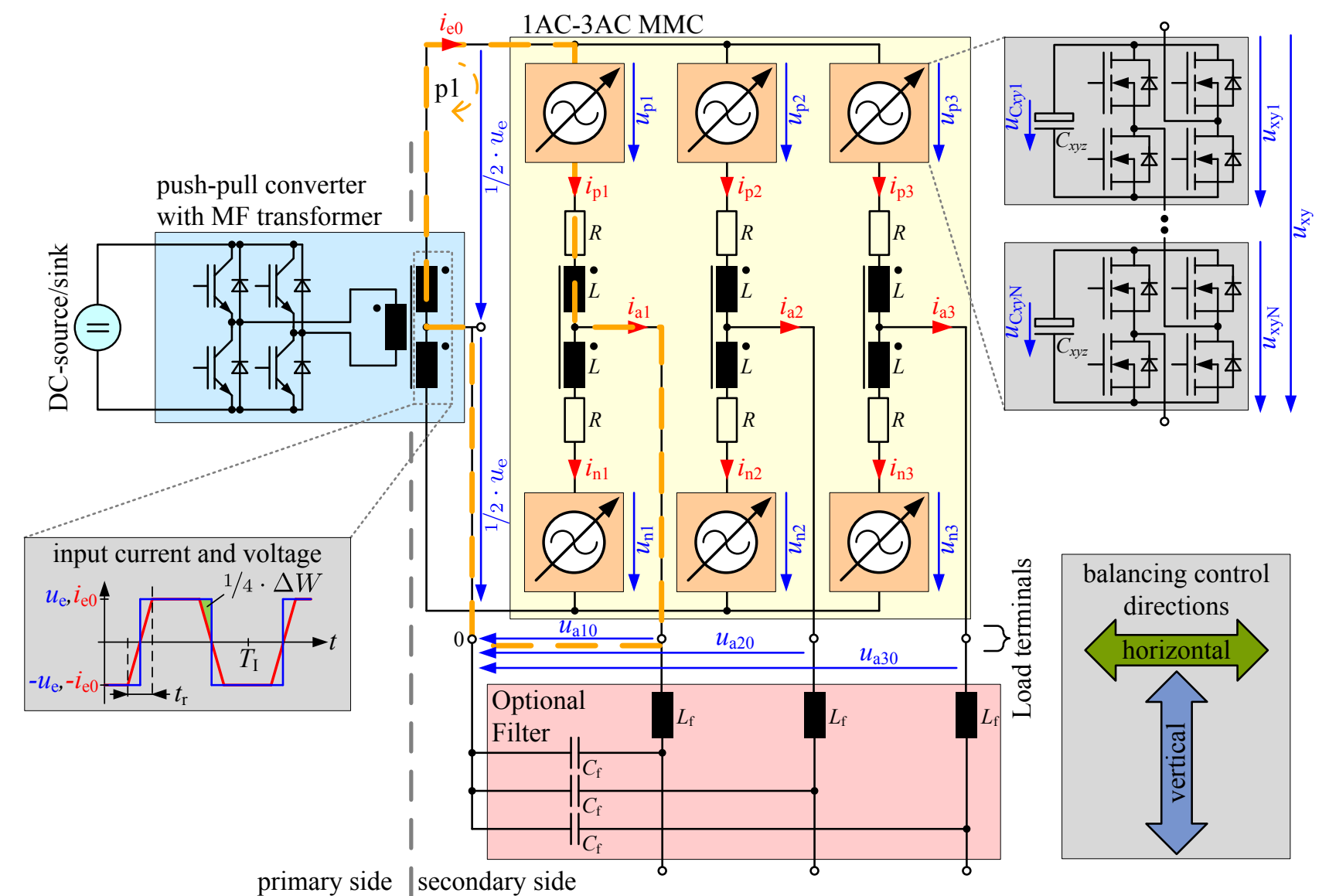

Fig. 1. Modular Multilevel Converter (MMC) with series connected full-bridge MMC cells fed by a push-pull converter and an optional output filter [1]

Therefore, switching losses are reduced which is a benefit. The MMC produces a variable three phase output voltage with adjustable amplitude and frequency. A low-pass filter can be installed at the output to further reduce the harmonics of the output voltage. The low-pass filter can be considered as a part of the load and is controlled using the output voltage of the MMC. Therefore, all derivations are made without a low-pass filter at the output.

The MMC consists of three phases [8]. Each phase has an upper arm $\mathrm{p}$, a lower arm $\mathrm{n}$ and a coupled inductor $L$ with a resistance $R$. Every arm consists of $N$ series-connected cells ( $z=$ cell number, $z \in\{\mathbb{N} \mid 1 \leq z \leq N\}$ ). All cells contain an H-bridge with a cell capacitor $C_{x y z}$ and can generate the voltages $0 \mathrm{~V},+u_{\mathrm{C} x y z},-u_{\mathrm{C} x y z}$ (upper or lower arm: $x \in\{p, n\}$, number of phase: $y \in\{1,2,3\})$. With this every arm is able to generate a variable arm voltage $u_{x y}$ within the following range:

$$
-u_{\mathrm{C} x y}=-\sum_{z=1}^{N} u_{\mathrm{C} x y z} \leq u_{x y} \leq \sum_{z=1}^{N} u_{\mathrm{C} x y z}=u_{\mathrm{C} x y}
$$

$u_{\mathrm{C} x y}$ is the arm capacitor voltage of the arm $x y$ which equals the sum of all cell capacitors in this arm. Each arm capacitor voltage can be converted to a corresponding arm energy $w_{\mathrm{C} x y}$, using

$$
w_{\mathrm{C} x y}=\frac{1}{2} C_{x y} \cdot u_{\mathrm{C} x y}^{2}
$$

with $C_{x y}=\frac{C_{x y z}}{N}$ as resulting arm capacitance. Since there are only capacitors and no power sources in each arm, the arm energy $w_{\mathrm{C} x y}$ can only be influenced by the arm power $p_{x y}=u_{x y} \cdot i_{x y}$. The arm energy $w_{\mathrm{C} x y}$ can be split into a constant average value $\bar{w}_{\mathrm{C}}$ and a time-dependent value $\tilde{w}_{\mathrm{C} x y}$ [8]:

$$
w_{\mathrm{C} x y}=\bar{w}_{\mathrm{C}}+\tilde{w}_{\mathrm{C} x y}=\bar{w}_{\mathrm{C}}+\int p_{x y} \mathrm{dt}
$$

The average of $p_{x y}$ must be zero to keep a constant average arm energy and, therefore, a constant arm capacitor voltage $u_{\mathrm{C} x y}$.

\section{A. Calculation of transformed equivalent circuits}

The analysis of the MMC as a decoupled system is described in this Section according to [8]. The analysis of the voltage loop (see orange dashed line in Fig. 1 for arm p1) for each phase is given by:

$$
\begin{aligned}
& -\frac{1}{2}\left[\begin{array}{l}
u_{\mathrm{e}} \\
u_{\mathrm{e}}
\end{array}\right]+\left[\begin{array}{l}
u_{\mathrm{p} y} \\
u_{\mathrm{n} y}
\end{array}\right]+L \cdot \frac{\mathrm{d}}{\mathrm{d} t}\left[\begin{array}{c}
1 / 2\left(i_{\mathrm{p} y}+i_{\mathrm{n} y}\right) \\
1 / 2\left(i_{\mathrm{p} y}+i_{\mathrm{n} y}\right)
\end{array}\right] \\
& +R \cdot\left[\begin{array}{c}
i_{\mathrm{p} y} \\
i_{\mathrm{n} y}
\end{array}\right]+\left[\begin{array}{c}
u_{\mathrm{a} y 0} \\
-u_{\mathrm{a} y 0}
\end{array}\right]=\left[\begin{array}{l}
0 \\
0
\end{array}\right]
\end{aligned}
$$

$u_{\mathrm{e}}$ is representing the input voltage of the MMC, $u_{x y}$ the arm voltage and $u_{\mathrm{a} y 0}$ the output voltage. Additionally, the arm 
currents $i_{x y}$ can be expressed as a superposition of the input current $i_{\mathrm{e} y}$ and the output current $i_{\mathrm{a} y}$.

$$
\left[\begin{array}{c}
i_{\mathrm{p} y} \\
i_{\mathrm{n} y}
\end{array}\right]=\left[\begin{array}{c}
i_{\mathrm{e} y} \\
i_{\mathrm{e} y}
\end{array}\right]+\frac{1}{2}\left[\begin{array}{c}
+i_{\mathrm{a} y} \\
-i_{\mathrm{a} y}
\end{array}\right]
$$

By using the amplitude invariant $\alpha \beta 0$-transformation matrix $C_{\alpha \beta 0}$ (6) and the definitions of eq. (7), the voltages and currents in (4) and (5) can be expressed for all six arms by the equations (8) and (9).

$$
\begin{gathered}
C_{\alpha \beta 0}=\left[\begin{array}{ccc}
2 / 3 & -1 / 3 & -1 / 3 \\
0 & 1 / \sqrt{3} & -1 / \sqrt{3} \\
1 / 3 & 1 / 3 & 1 / 3
\end{array}\right] \\
{\left[\begin{array}{l}
u_{\alpha} \\
u_{\beta} \\
u_{0}
\end{array}\right]=C_{\alpha \beta 0} \cdot\left[\begin{array}{l}
u_{1} \\
u_{2} \\
u_{3}
\end{array}\right], \quad\left[\begin{array}{c}
i_{\alpha} \\
i_{\beta} \\
i_{0}
\end{array}\right]=C_{\alpha \beta 0} \cdot\left[\begin{array}{c}
i_{1} \\
i_{2} \\
i_{3}
\end{array}\right]}
\end{gathered}
$$

The eq. (8) and (9) are describing the decoupled MMC in the $\alpha \beta 0$-transformed system. With this representation the currents concerning the MMC control $i_{\mathrm{e} \alpha / \beta / 0}$ can be controlled independently from the output voltage $u_{\mathrm{a} \alpha / \beta / 0}$. The currents $i_{\mathrm{e} \alpha / \beta / 0}$ are influenced by the inductor voltages $u_{\mathrm{L} \alpha / \beta / 0}$.

$$
\begin{gathered}
2 R \cdot\left[\begin{array}{c}
i_{\mathrm{e} \alpha} \\
i_{\mathrm{e} \beta} \\
i_{\mathrm{e} 0}
\end{array}\right]+2 L \cdot \frac{\mathrm{d}}{\mathrm{dt}}\left[\begin{array}{c}
i_{\mathrm{e} \alpha} \\
i_{\mathrm{e} \beta} \\
1 / 3 \cdot i_{\mathrm{e} 0}
\end{array}\right] \\
=\left[\begin{array}{c}
u_{\mathrm{L} \alpha} \\
u_{\mathrm{L} \beta} \\
u_{\mathrm{L} 0}
\end{array}\right]=\left[\begin{array}{c}
-u_{\mathrm{p} \alpha}-u_{\mathrm{n} \alpha} \\
-u_{\mathrm{p} \beta}-u_{\mathrm{n} \beta} \\
+u_{\mathrm{e}}-u_{\mathrm{p} 0}-u_{\mathrm{n} 0}
\end{array}\right] \\
{\left[\begin{array}{c}
u_{\mathrm{a} \alpha} \\
u_{\mathrm{a} \beta} \\
u_{\mathrm{a} 0}
\end{array}\right]=\frac{1}{2} \cdot\left[\begin{array}{c}
-u_{\mathrm{p} \alpha}+u_{\mathrm{n} \alpha} \\
-u_{\mathrm{p} \beta}+u_{\mathrm{n} \beta} \\
-u_{\mathrm{p} 0}+u_{\mathrm{n} 0}
\end{array}\right]-\frac{1}{2} R \cdot\left[\begin{array}{c}
i_{\mathrm{a} \alpha} \\
i_{\mathrm{a} \beta} \\
i_{\mathrm{a} 0}
\end{array}\right]}
\end{gathered}
$$

In Fig. 2 the equations (8) and (9) are represented as equivalent circuits for the e-currents and a-voltages for a decoupled control of the MMC. The e-currents are used to balance the arm energies and also can be used to reduce energy pulsations in the arm capacitors. The current $i_{\mathrm{e} 0}$ is the input current of the MMC. The currents $i_{\mathrm{e} \alpha / \beta}$ are inner currents and do not appear at the input side nor the output side.

\section{B. Arm power analysis}

The occurring arm powers are analyzed in this Section. Each instantaneous arm power can be calculated by using $p_{x y}=u_{x y} \cdot i_{x y}$. For example, the calculation of the arm power $p_{\mathrm{p} 1}$ results in (neglecting the voltage $u_{\mathrm{L} 1}$ and the resistance $R$ ):

$$
p_{\mathrm{p} 1}=u_{\mathrm{p} 1} \cdot i_{\mathrm{p} 1}=\left(\frac{1}{2} \cdot u_{\mathrm{e}}-u_{\mathrm{a} 10}\right) \cdot\left(i_{\mathrm{e} 1}+\frac{1}{2} \cdot i_{\mathrm{a} 1}\right)
$$

Eq. (10) shows that the arm power is composed of multiple parts of different voltages and currents. The equation can be written down for all six arms. To simplify the representation, as shown in [8], a mean value $p_{\Sigma y}$ and a difference value $p_{\Delta y}$ of the power components is introduced in eq. (11). The mean power affects the upper and lower arm power with the same signs and, therefore, increases or decreases the arm energy in the according phase. The difference value affects the upper and lower arm power with different signs and, therefore, shifts energy between the upper and lower arm of the according phase.

$$
p_{\Sigma y}=\frac{1}{2}\left(p_{\mathrm{p} y}+p_{\mathrm{n} y}\right), \quad p_{\Delta y}=\left(p_{\mathrm{p} y}-p_{\mathrm{n} y}\right)
$$

a)

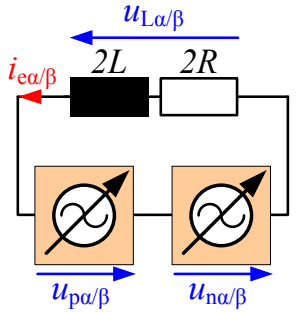

b)

Load terminal

(without filter)
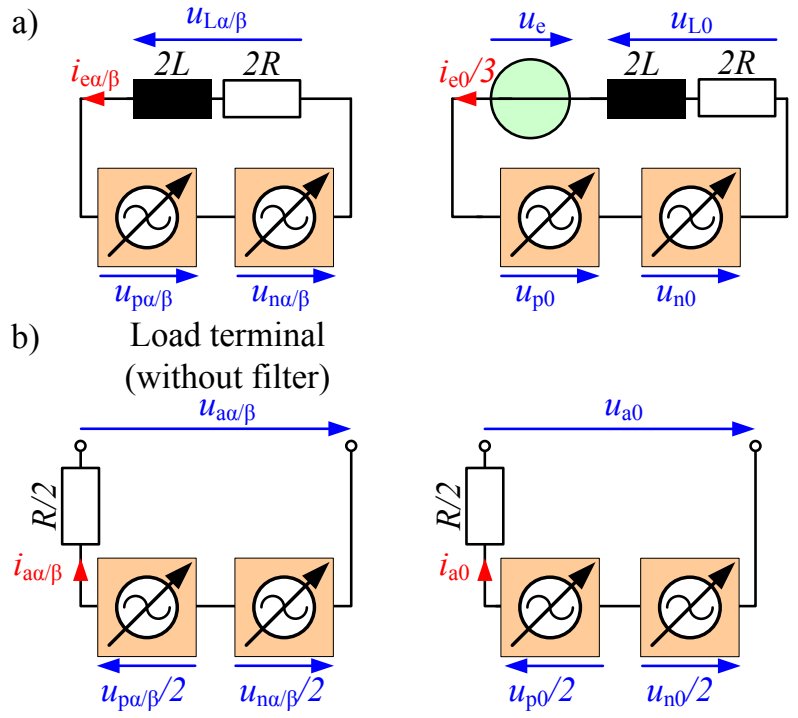

Fig. 2. Resulting equivalent circuits of the transformed e-currents (a) and a-currents and a-voltages (b) [8]

Eq. (11) are $\alpha \beta 0$-transformed into $p_{\Sigma \alpha / \beta / 0}$ and $p_{\Delta \alpha / \beta / 0}$ using eq. (12) and (13) to simplify energy control and balancing.

$$
\begin{aligned}
& {\left[\begin{array}{c}
p_{\Sigma \alpha} \\
p_{\Sigma \beta} \\
p_{\Sigma 0}
\end{array}\right]=C_{\alpha \beta 0} \cdot\left[\begin{array}{l}
p_{\Sigma 1} \\
p_{\Sigma 2} \\
p_{\Sigma 3}
\end{array}\right]} \\
& {\left[\begin{array}{c}
p_{\Delta \alpha} \\
p_{\Delta \beta} \\
p_{\Delta 0}
\end{array}\right]=C_{\alpha \beta 0} \cdot\left[\begin{array}{l}
p_{\Delta 1} \\
p_{\Delta 2} \\
p_{\Delta 3}
\end{array}\right]}
\end{aligned}
$$

The expression $p_{\Sigma \alpha / \beta / 0}$ is also referred as horizontal direction and shifts energy between the three phases. $p_{\Delta \alpha / \beta / 0}$ is referred as vertical direction and shifts energy between the upper and lower arms (see Fig. 1). For a simplified representation the output voltages and output currents were defined as a polar coordinate system according to eq. (14)-(17). They describe a symmetric and sinusoidal output voltage $u_{\mathrm{a}}$ and output current $i_{\mathrm{a}}$ with the output angle $\gamma_{\mathrm{A}}=\omega_{\mathrm{A}} \cdot t$ and a phase shift $\varphi_{\mathrm{a}}$ between output voltage and output current:

$$
\begin{aligned}
u_{\mathrm{a} \alpha} & =\hat{u}_{\mathrm{a}} \cdot \cos \left(\gamma_{\mathrm{a}}\right) \\
u_{\mathrm{a} \beta} & =\hat{u}_{\mathrm{a}} \cdot \sin \left(\gamma_{\mathrm{a}}\right) \\
i_{\mathrm{a} \alpha} & =\hat{i}_{\mathrm{a}} \cdot \cos \left(\gamma_{\mathrm{a}}-\varphi_{\mathrm{a}}\right) \\
i_{\mathrm{a} \beta} & =\hat{i}_{\mathrm{a}} \cdot \sin \left(\gamma_{\mathrm{a}}-\varphi_{\mathrm{a}}\right)
\end{aligned}
$$

Furthermore, eq. (18)-(20) are used to describe the e-currents $i_{\mathrm{e} \alpha / \beta / 0}$. The currents $i_{\mathrm{eH} \alpha / \beta / 0}$ are used for controlling the horizontal direction. The vertical direction is influenced by the currents $\hat{i}_{\mathrm{eVp}}$ and $\hat{i}_{\mathrm{eVn}}$ with the angle $\gamma_{\mathrm{e}}$ and phase shift $\varphi_{\mathrm{e}}$. $\hat{i}_{\mathrm{eVp}}$ represents a positive sequence, $\hat{i}_{\mathrm{eVn}}$ represents a negative sequence.

$$
\begin{aligned}
& i_{\mathrm{e} \alpha}=i_{\mathrm{eH} \alpha} \\
& +\hat{i}_{\mathrm{eVp}} \cdot \cos \left(\gamma_{\mathrm{e}}\right)+\hat{i}_{\mathrm{eVn}} \cdot \cos \left(-\gamma_{\mathrm{e}}+\varphi_{\mathrm{e}}\right) \\
& i_{\mathrm{e} \beta}=i_{\mathrm{eH} \beta} \\
& +\hat{i}_{\mathrm{eVp}} \cdot \sin \left(\gamma_{\mathrm{e}}\right)+\hat{i}_{\mathrm{eVn}} \cdot \sin \left(-\gamma_{\mathrm{e}}+\varphi_{\mathrm{e}}\right) \\
& i_{\mathrm{e} 0}=i_{\mathrm{eH} 0}
\end{aligned}
$$




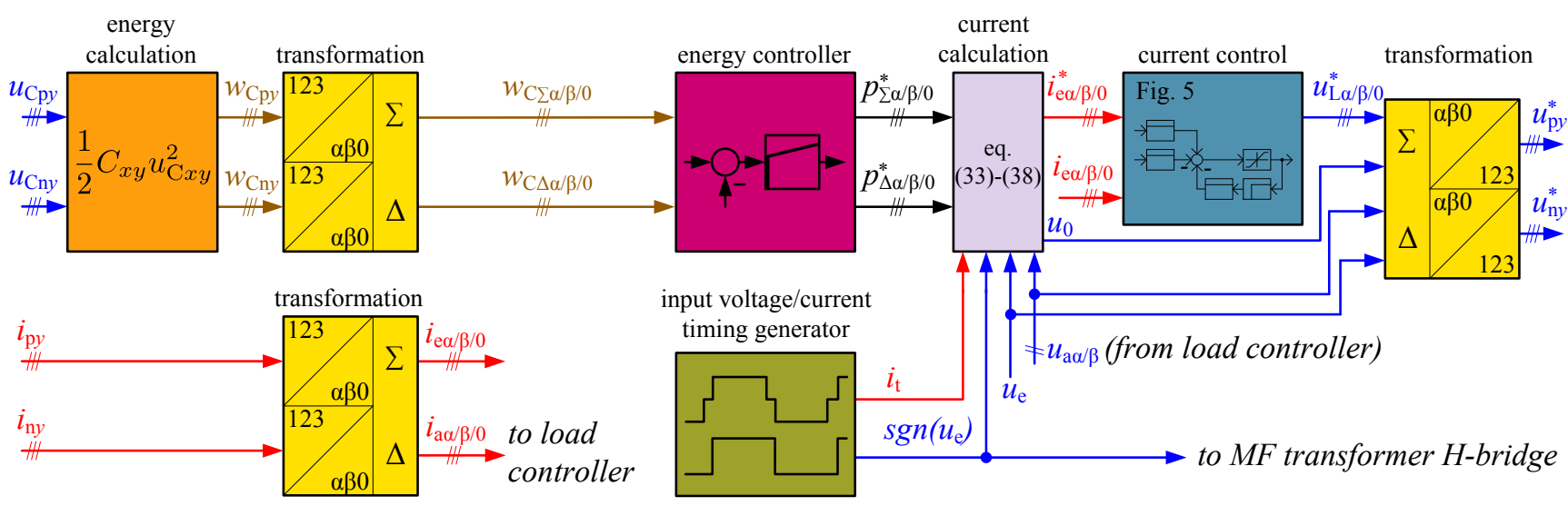

Fig. 3. Overall cascaded control scheme for MMC with a square-wave input voltage

The complete transformed arm power equations are shown in the eq. (48)-(53) in the Appendix. According to eq. (3) the arm energies are controlled with an active power component at the arm capacitors. A reactive power causes only an oscillation of the arm energies. Therefore, $p_{\Sigma \alpha / \beta / 0}$ and $p_{\Delta \alpha / \beta / 0}$ must be searched through for controllable terms. The following values are fixed by the application and not controllable: $u_{\mathrm{e}}, \hat{u}_{\mathrm{a}}, \hat{i}_{\mathrm{a}}, \gamma_{\mathrm{a}}$ and $\varphi_{\mathrm{a}}$. The remaining values can be controlled independently as shown in the last chapter: $i_{\mathrm{e} \alpha}, i_{\mathrm{e} \beta}, i_{\mathrm{e} 0}$ and $u_{\mathrm{a} 0}$. Preferably, $u_{\mathrm{a} 0}$ should not be used to balance energies because under some circumstances it is possible that a load is connected with its star point at the transformer center tap. The following terms are chosen to generate active power at the arm capacitors:

$$
\begin{aligned}
& \text { (48a) : } \quad p_{\Sigma \alpha, \mathrm{b}}=1 / 2 \cdot u_{\mathrm{e}} i_{\mathrm{eH} \alpha} \\
& \text { (49a) : } \quad p_{\Sigma \beta, \mathrm{b}}=1 / 2 \cdot u_{\mathrm{e}} i_{\mathrm{eH} \beta} \\
& \text { (50a) : } \quad p_{\Sigma 0, \mathrm{~b}}=1 / 2 \cdot u_{\mathrm{e}} i_{\mathrm{eH} 0} \\
& \text { (51b) : } \quad p_{\Delta \alpha, \mathrm{b}}=+\hat{u}_{\mathrm{a}} \hat{i}_{\mathrm{eVn}} \cdot \sin \left(-\gamma_{\mathrm{e}}+\varphi_{\mathrm{e}}\right) \cdot \sin \left(\gamma_{\mathrm{a}}\right) \\
& \text { (51c) : } \quad-\hat{u}_{\mathrm{a}} \hat{i}_{\mathrm{eVn}} \cdot \cos \left(-\gamma_{\mathrm{e}}+\varphi_{\mathrm{e}}\right) \cdot \cos \left(\gamma_{\mathrm{a}}\right) \\
& \text { (52b) : } \quad p_{\Delta \beta, \mathrm{b}}=+\hat{u}_{\mathrm{a}} \hat{i}_{\mathrm{eVn}} \cdot \sin \left(-\gamma_{\mathrm{e}}+\varphi_{\mathrm{e}}\right) \cdot \cos \left(\gamma_{\mathrm{a}}\right) \\
& \text { (52c) : } \quad+\hat{u}_{\mathrm{a}} \hat{i}_{\mathrm{eVn}} \cdot \cos \left(-\gamma_{\mathrm{e}}+\varphi_{\mathrm{e}}\right) \cdot \sin \left(\gamma_{\mathrm{a}}\right) \\
& \text { (53a): } \quad p_{\Delta 0, \mathrm{~b}}=-\hat{u}_{\mathrm{a}} \hat{i}_{\mathrm{eVp}} \cdot \cos \left(\gamma_{\mathrm{e}}\right) \cdot \cos \left(\gamma_{\mathrm{a}}\right) \\
& \text { (53b) : } \\
& -\hat{u}_{\mathrm{a}} \hat{i}_{\mathrm{eVp}} \cdot \sin \left(\gamma_{\mathrm{e}}\right) \cdot \sin \left(\gamma_{\mathrm{a}}\right)
\end{aligned}
$$

According to Eq. (21) to (26), power in each direction can be generated by using one of the currents $i_{\mathrm{eH} \alpha / \beta / 0}$ or the currents $\hat{i}_{\mathrm{eVp} / \mathrm{n}}$ with the angle $\gamma_{\mathrm{e}}$ with the appropriate voltage. To achieve a horizontal power, the currents $i_{\mathrm{eH} \alpha / \beta / 0}$ must be in phase and have the same frequency as the input voltage $u_{\mathrm{e}}$ and, therefore, should be a square-wave. Vertical active power is produced with the currents $\hat{i}_{\mathrm{eVp} / \mathrm{n}}$ and an angle of $\gamma_{\mathrm{e}}=\gamma_{\mathrm{a}}$. Simplifying eq. (24)-(26) with $\gamma_{\mathrm{e}}=\gamma_{\mathrm{a}}$ results in:

$$
\begin{aligned}
p_{\Delta \alpha, \mathrm{b}} & =+\hat{u}_{\mathrm{a}} \hat{i}_{\mathrm{eVn}} \cdot\left(-\cos \varphi_{e}\right) \\
p_{\Delta \beta, \mathrm{b}} & =+\hat{u}_{\mathrm{a}} \hat{i}_{\mathrm{eVn}} \cdot \sin \varphi_{e} \\
p_{\Delta 0, \mathrm{~b}} & =-\hat{u}_{\mathrm{a}} \hat{i}_{\mathrm{eVp}}
\end{aligned}
$$

The direction of the vertical power $p_{\Delta \alpha, b / \beta, b}$ is determined by the phase shift $\varphi_{\mathrm{e}}$.

\section{CASCADED CONTROL SCHEME}

Fig. 3 shows the cascaded control scheme which is derived from the arm power analysis in Section II-B. First, the arm energies $w_{\mathrm{C} x y}$ are calculated from the measured arm capacitor voltages $u_{\mathrm{C} x y}$ according to (2). Then the arm energies $w_{\mathrm{C} x y}$ and the measured arm currents $i_{x y}$ are $\alpha \beta 0$-transformed.

The arm energies are fed to the superposed energy controller which calculates the requested power for each of the six components $\left(p_{\Sigma \alpha / \beta / 0}^{*}\right.$ and $\left.p_{\Delta \alpha / \beta / 0}^{*}\right)$. In Fig. 4 the control loop for the energy controllers is shown. It consists of a dead time $T_{\mathrm{t}}$ from the subordinated current controller and an integrator with a gain of 1 . An optional filter $T_{\mathrm{f}}$ can be placed in the feedback loop for damping the AC part of the energy oscillations. A PI controller is chosen to control the energy and is designed by using the symmetrical optimum according to the formulas (30)(32) with $T_{\sigma \mathrm{w}}$ as total of the minor time constants. $a$ describes the damping factor of the closed loop control system and is chosen to 2 [9].

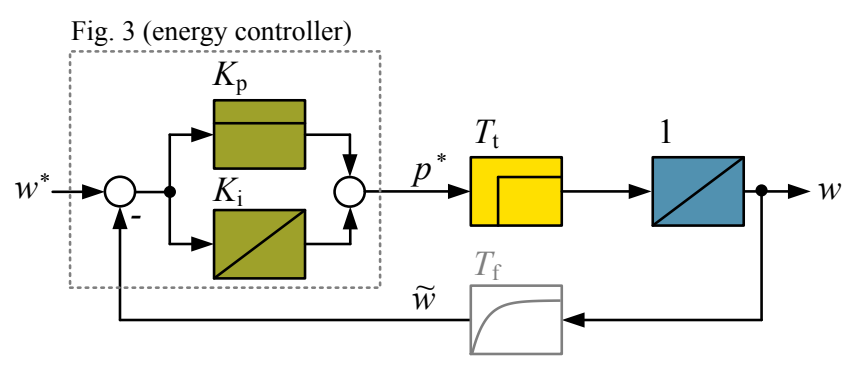

Fig. 4. Resulting control loop for superposed energy controller

$$
\begin{aligned}
T_{\sigma \mathrm{w}} & =T_{\mathrm{t}}+T_{\mathrm{f}} \\
K_{\mathrm{p}} & =\frac{1}{T_{\sigma \mathrm{w}} \cdot a} \\
K_{\mathrm{i}} & =\frac{1}{T_{\sigma \mathrm{w}}^{2} \cdot a^{3}}
\end{aligned}
$$

All reference values for the energy controllers are chosen to 0 except for $w_{\mathrm{C} \Sigma 0}^{*}=\bar{w}_{\mathrm{C}}$ to accomplish a symmetrical distribution of the energies.

In the next step the power reference values are converted to current reference values $i_{\mathrm{e} \alpha / \beta / 0}^{*}$ for the subordinated current 
controllers. Therefore, eq. (21)-(23) and (27)-(29) are solved for the according current respectively angle:

$$
\begin{aligned}
i_{\mathrm{eH} \alpha} & =\frac{2}{u_{\mathrm{e}}} \cdot p_{\Sigma \alpha, \mathrm{b}} \\
i_{\mathrm{eH} \beta} & =\frac{2}{u_{\mathrm{e}}} \cdot p_{\Sigma \beta, \mathrm{b}} \\
i_{\mathrm{eH} 0} & =\frac{2}{u_{\mathrm{e}}} \cdot p_{\Sigma 0, \mathrm{~b}} \\
\hat{i}_{\mathrm{eVn}} & =\frac{1}{\hat{u}_{\mathrm{a}}} \cdot \sqrt{p_{\Delta \alpha, \mathrm{b}}^{2}+p_{\Delta \beta, \mathrm{b}}^{2}} \\
\varphi_{\mathrm{e}} & =\arctan \frac{p_{\Delta \beta, \mathrm{b}}}{-p_{\Delta \alpha, \mathrm{b}}} \\
\hat{i}_{\mathrm{eVp}} & =\frac{1}{\hat{u}_{\mathrm{a}}} \cdot p_{\Delta 0, \mathrm{~b}}
\end{aligned}
$$

Horizontal active power is generated by using the input voltage $u_{\mathrm{e}}$ and one of the currents $i_{\mathrm{eH} \alpha / \beta / 0}$. Vertical active power is achieved by using the output voltage $u_{\mathrm{a} \alpha / \beta}$ and a positive or negative sequence current $i_{\mathrm{eVp} / \mathrm{n}}$. For each power component one current component is calculated and all current components must be summed up to obtain the reference value for the current controller according to eq. (18)-(20).

The current $i_{\mathrm{eH} \alpha / \beta / 0}$ cannot be an ideal square-wave because the controlled system is an RL load (see Fig. 2a). Therefore, the current $i_{\mathrm{eH} \alpha / \beta / 0}$ is trapezoidal and that fact reduces the active power, resulting in a lower energy transferred, shown as $\Delta W$ in Fig. 1. $T_{\mathrm{I}}$ is the period of the input frequency, $t_{\mathrm{r}}$ is the duration for reversing the current $i_{\mathrm{e} 0}$. The according components of the e-currents which are generated by the input voltage $u_{\mathrm{e}}$ are multiplied by the correction factor $\xi_{\mathrm{T}}$ :

$$
\xi_{\mathrm{T}}=\frac{T_{\mathrm{I}}}{T_{\mathrm{I}}-t_{\mathrm{r}}}
$$

To achieve zero current switching (ZCS) the input voltage $u_{\mathrm{e}}$ and input current $i_{\mathrm{e} 0}$ have to be synchronized. For this purpose a control block is introduced which modifies the reference value for the input current using $i_{\mathrm{t}}$ to a trapezoidal shape, see Fig. 3. The reference value is for one control period 0 to generate an input current of $0 \mathrm{~A}$. Then the zero current switching (ZCS) of the push-pull-converter H-bridge occurs at the end of a control period. Simultaneously the input voltage at the MF-transformer is switched. ZCS at the primary side $\mathrm{H}-$ bridge is achieved when the magnetizing current is delivered from the MMC side of the transformer. It must be pointed out that the synchronization of the input voltage $u_{\mathrm{e}}$ and the MMC voltage $u_{\mathrm{p} / \mathrm{n} 0}$ (see Fig. 2a) is crucial. Due to the low arm inductor $L$ a displacement between $u_{\mathrm{e}}$ and $u_{\mathrm{p} / \mathrm{n} 0}$ during the voltage reversal results in an unwanted increase of the input current $i_{\mathrm{e} 0}$.

The current controller is fed by the reference values $i_{\mathrm{e} \alpha / \beta / 0}^{*}$ and the actual current values $i_{\mathrm{e} \alpha / \beta / 0}$. Due to the fact that e.g. $p_{\Sigma 0}$ is generated using $u_{\mathrm{e}} \cdot i_{\mathrm{e} 0}$ and $u_{\mathrm{e}}$ is a square-wave voltage, $i_{\mathrm{e} 0}$ is controlled as a trapezoidal current. Therefore, the current controller needs a very high dynamic. The current controller is designed as a dead-beat controller [10], see Fig. 5.

A dead-beat controller was chosen because a continuously designed and in digital domain implemented PI-controller is too slow, especially when the frequency of the input voltages

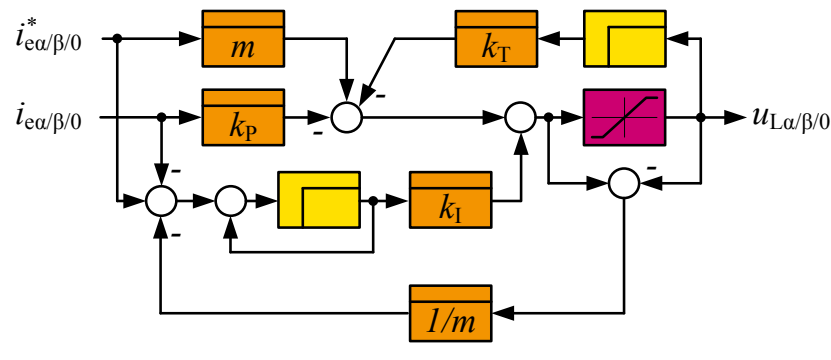

Fig. 5. Dead-beat current controller [10]

comes to the same order as the control frequency. The controlled system for each current $i_{\mathrm{e} \alpha / \beta / 0}$ is an RL load (see Fig. 2a). The controller is identical for all three currents if the input voltage is fed-forward (see Fig. 3 and Fig. 5). The current controller is parameterized with the following equations (40)(45). $T_{\mathrm{A}}$ represents the control period, $\tau_{\mathrm{w}}$ is the time constant of the closed control loop and is chosen to $0 \mathrm{~s}$ to achieve deadbeat behavior. $\tau_{\mathrm{I}}$ is the integration time constant and is chosen to $10 \mathrm{~ms}$. The integrator is used to eliminate any DC offsets in the currents. The load is considered using the resistance $R$ and the time constant of the load $\tau=L / R$. The resulting dead-time of the closed-loop current control is chosen to $T_{\mathrm{t}}=2 \cdot T_{\mathrm{A}}$.

$$
\begin{aligned}
z_{\mathrm{w}}= & \mathrm{e}^{-\frac{T_{\mathrm{A}}}{\tau_{\mathrm{w}}}} \\
z_{\mathrm{I}}= & \mathrm{e}^{-\frac{T_{\mathrm{A}}}{\tau_{\mathrm{I}}}} \\
m= & \left(1-z_{\mathrm{w}}\right) \cdot \frac{R}{1-\mathrm{e}^{-\frac{T_{\mathrm{A}}}{\tau}}} \\
k_{\mathrm{I}}= & \left(1-z_{\mathrm{I}}\right) \cdot m \\
k_{\mathrm{T}}= & 1-z_{\mathrm{w}}-z_{\mathrm{I}}+\mathrm{e}^{-\frac{T_{\mathrm{A}}}{\tau}} \\
k_{P}= & \left(\left(1-z_{\mathrm{w}}\right) \cdot\left(1-z_{\mathrm{I}}\right)\right. \\
& \left.+\left(1-z_{\mathrm{w}}-z_{\mathrm{I}}+e^{-\frac{T_{\mathrm{A}}}{\tau}}\right) \cdot e^{-\frac{T_{\mathrm{A}}}{\tau}}\right) \\
& \cdot \frac{R}{1-e^{-\frac{T_{\mathrm{A}}}{\tau}}}
\end{aligned}
$$

The final step is the back transformation of the voltages into the 123-system. The resulting voltages to control the MMC are sent to a modulator which balances and selects the according cells in the six converter arms [8]. The polarity of the input voltage $u_{\mathrm{e}}$ is sent to the modulator of the H-Bridge.

\section{Simulation}

This Section presents the simulation results of the proposed topology using MATLAB/SIMULINK. In the simulation the MF transformer was replaced by a controlled square-wave voltage source. Each arm was modeled as a variable voltage source to reduce simulation time. Therefore, a model for the arm capacitor voltage is implemented. The following parameters were chosen for the simulation (see Table I):

Fig. 6 shows simulation results of the proposed current control concept for the square-wave powered Modular Multilevel Converter under full load. The output power is about $52 \mathrm{~kW}$. As it can be seen, the dead-beat controller works properly and reverses the input current during two control periods according to the current reference. The input current and input voltage are completely synchronized and, therefore, only active power 
TABLE I. SIMUlation PARAMETER

\begin{tabular}{ccl} 
Symbol & Value & \multicolumn{1}{c}{ Description } \\
\hline \hline$N$ & 8 & cells per arm \\
$C_{x y z}$ & $4400 \mu \mathrm{F}$ & cell capacitance \\
$u_{\mathrm{C} x y}^{*}$ & $800 \mathrm{~V}$ & set point arm capacitor voltage \\
$\hat{U}_{\mathrm{e}}$ & $700 \mathrm{~V}$ & input voltage \\
\hline$L$ & $100 \mu \mathrm{H}$ & arm inductance \\
$R$ & $10 \mathrm{~m} \Omega$ & arm resistance \\
\hline $1 / T_{\mathrm{I}}$ & $1.25 \mathrm{kHz}$ & input frequency \\
$1 / T_{\mathrm{A}}$ & $10 \mathrm{kHz}$ & control frequency \\
$1 / T_{\mathrm{f}}$ & $10 \mathrm{~Hz}$ & cut-off frequency for feedback filter at energy controller
\end{tabular}
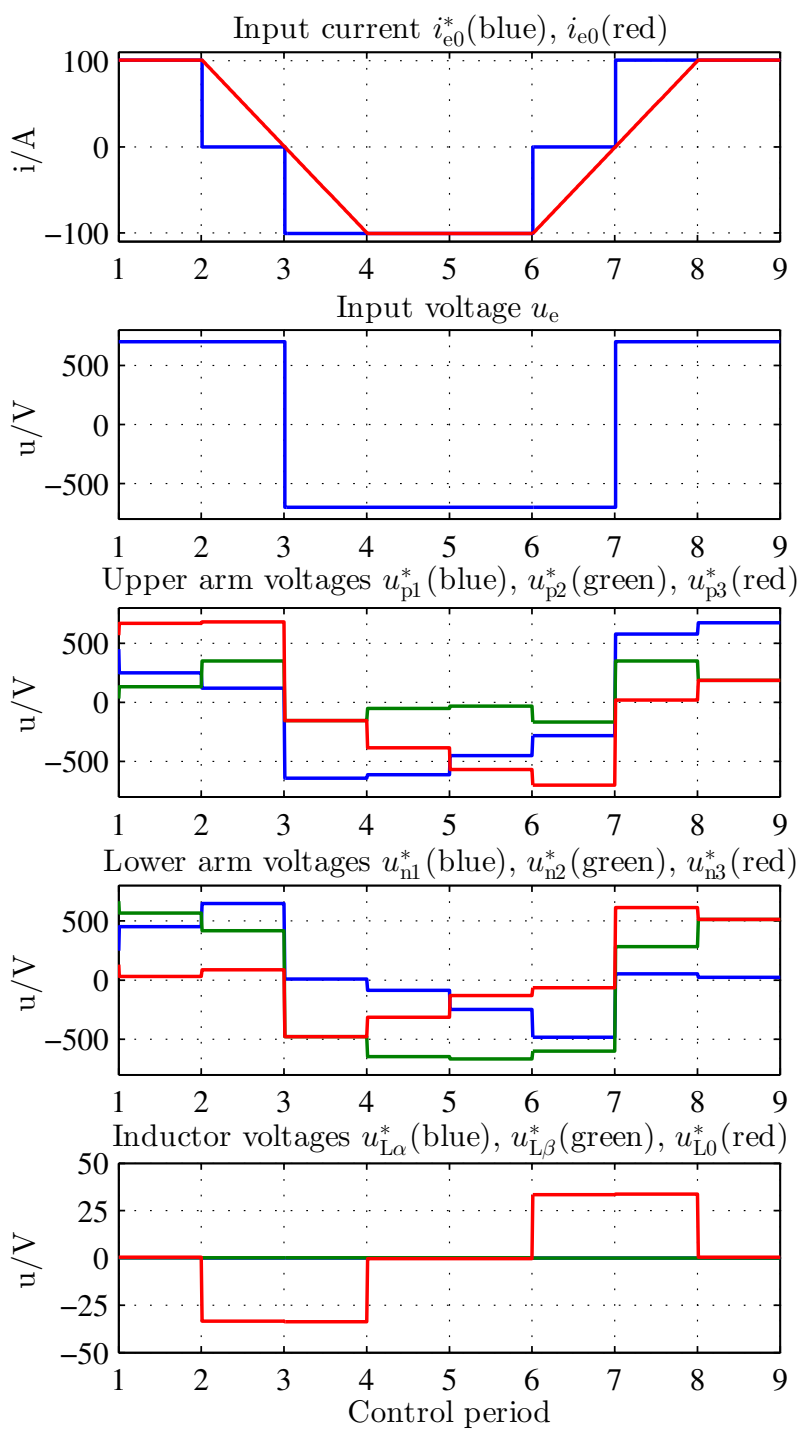

Fig. 6. Simulation results of the subordinated current controller during one period of the input voltage $\hat{u}_{\mathrm{e}}=700 \mathrm{~V}$ and peak input current $\hat{i}_{\mathrm{e} 0}=100 \mathrm{~A}$. Output amplitude is chosen to $\hat{u}_{\mathrm{a}}=325 \mathrm{~V}$ and the output frequency is chosen to $f_{\mathrm{a}}=1 \mathrm{kHz}$. is transferred. It must be noted that the demanded arm voltages $u_{x y}^{*}$ do never exceed the maximum arm voltage $u_{\mathrm{C} x y}^{*}$. This is important for current reversal because if this is not ensured the input current $i_{\mathrm{e} 0}$ would not be zero at the end of the control period and, therefore, no ZCS at the H-bridge could be achieved. The inductor voltage $u_{\mathrm{L} 0}^{*}$ shows that the current reversal under full load needs only about $35 \mathrm{~V}$ and is small compared to the arm capacitor voltage.

Fig. 7 shows an output frequency sweep from $-1000 \mathrm{~Hz}$ to $+1000 \mathrm{~Hz}$. As load a 3-phase resistor was chosen, hence no phase shift between output voltage $u_{\mathrm{a} y}$ and output current $i_{\mathrm{a} y}$ can be observed. It must be pointed out, that the capacitor voltages are balanced over the complete output frequency range properly. No additional balancing action is needed for an output frequency of $0 \mathrm{~Hz}$ due to the fact the input frequency is an $\mathrm{AC}$ voltage. Between an output frequency of $-300 \mathrm{~Hz}$ to $+300 \mathrm{~Hz}$ a compensation to reduce energy pulsations in the arm capacitors is enabled [8]. The responsible terms for high energy pulsations in the arm capacitor voltages at low output frequencies are eq. (48b) and (49b). Assuming $\gamma_{\mathrm{a}}=\omega_{\mathrm{a}} \cdot t$ the power terms have a low frequency when the output frequency is low. According to eq. (3) the amplitude of the reactive power is proportional to $1 / \omega_{\mathrm{a}}$. To eliminate the energy pulsations the opposed powers from eq. (48b) and (49b) are used:

$$
\begin{aligned}
& p_{\Sigma \alpha, \mathrm{LF}}=+\frac{1}{4} \hat{u}_{\mathrm{a}} \hat{i}_{\mathrm{a}} \cos \left(2 \cdot \gamma_{\mathrm{a}}-\varphi_{\mathrm{a}}\right) \\
& p_{\Sigma \beta, \mathrm{LF}}=-\frac{1}{4} \hat{u}_{\mathrm{a}} \hat{i}_{\mathrm{a}} \sin \left(2 \cdot \gamma_{\mathrm{a}}-\varphi_{\mathrm{a}}\right)
\end{aligned}
$$

The currents are calculated using eq. (33) and (34). An increase of the arm currents in the according output frequency range can be observed.

\section{CONCLUSION}

In this paper a control concept for a Modular Multilevel Converter (MMC) fed by a push-pull converter via a medium frequency transformer is introduced. The advantages of the topology are galvanic isolation of the MMC, no limitation of the output power at DC output voltage and a reduced transformer size and cell capacitance. Due to the push-pull converter the input voltage of the MMC is a medium frequency square-wave voltage. To control the required medium frequency trapezoidal input current, a subordinated dead-beat current controller for the MMC is introduced. The current reference values are calculated with the help of the transformed arm power analysis. The transformed arm power reference values are obtained from superposed PI controllers which performs the balancing of the arm energies. The excellent performance of the presented cascaded control scheme is demonstrated in a simulation. A prototype with $50 \mathrm{~kW}$ output power is under construction and will be used for power grid emulations, Power Hardware-in-the-Loop Emulators and testing and measurement of electrical components.

\section{APPENDIX}

Complete transformed arm power equations for the squarewave powered $1 \mathrm{AC}-3 \mathrm{AC}$ MMC:

$$
p_{\Sigma \alpha}=+\frac{1}{2} u_{\mathrm{e}} i_{\mathrm{eH} \alpha}
$$



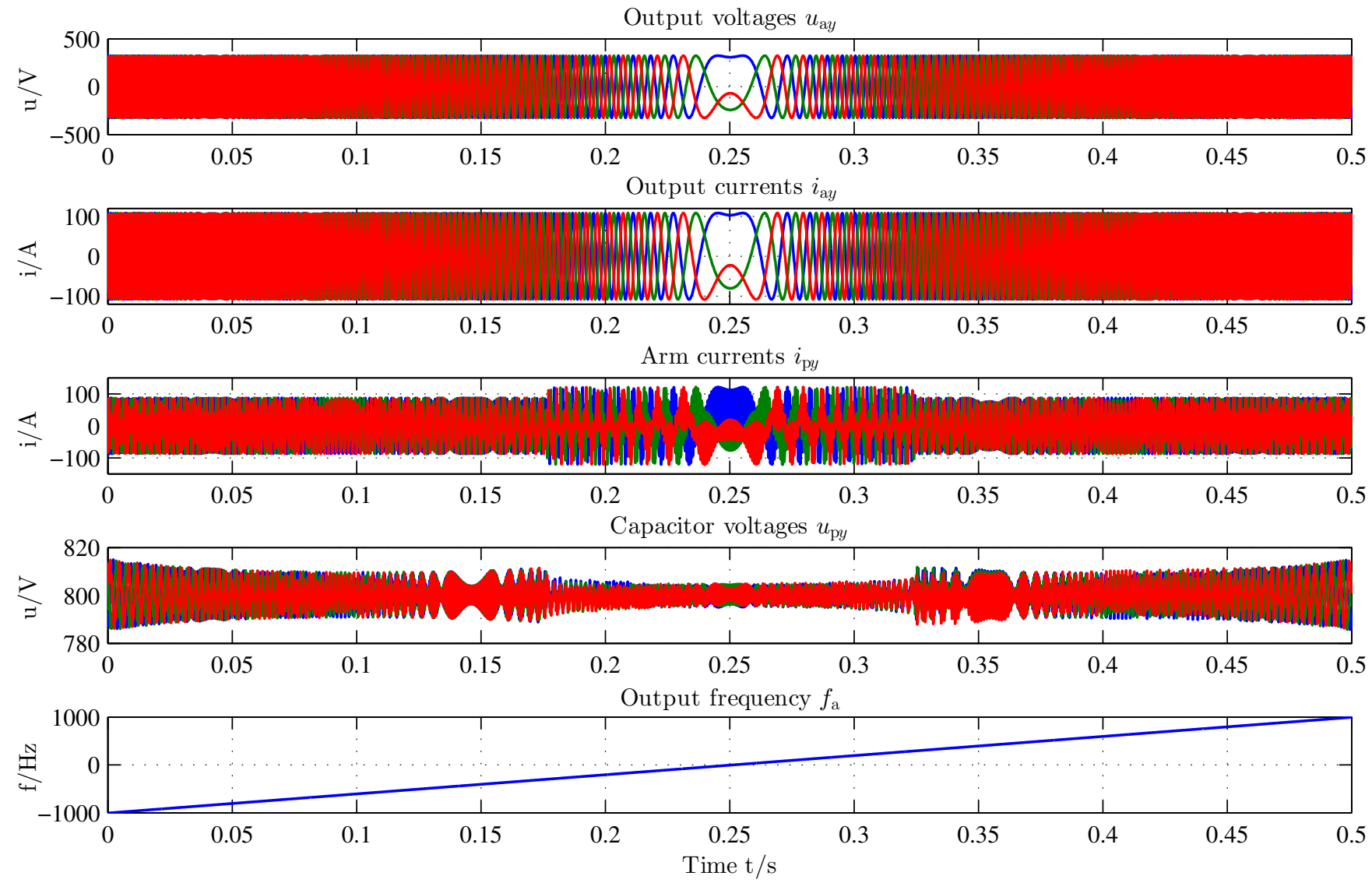

Fig. 7. Simulation results of proposed control concept with an output frequency sweep from $-1000 \mathrm{~Hz}$ to $1000 \mathrm{~Hz}$. Output amplitude is chosen to $\hat{u}_{\mathrm{a}}=325 \mathrm{~V}$

$$
\begin{aligned}
& -\frac{1}{4} \hat{u}_{\mathrm{a}} \hat{i}_{\mathrm{a}} \cdot \cos \left(2 \cdot \gamma_{\mathrm{a}}-\varphi_{\mathrm{a}}\right) \\
& -\frac{1}{2} u_{\mathrm{a} 0} \hat{i}_{\mathrm{a}} \cdot \cos \left(\gamma_{\mathrm{a}}-\varphi_{\mathrm{a}}\right) \\
& +\frac{1}{2} u_{\mathrm{e}} \hat{i}_{\mathrm{eVp}} \cdot \cos \left(\gamma_{\mathrm{e}}\right) \\
& +\frac{1}{2} u_{\mathrm{e}} \hat{i}_{\mathrm{eVn}} \cdot \cos \left(-\gamma_{\mathrm{e}}+\varphi_{\mathrm{e}}\right) \\
p_{\Sigma \beta}= & +\frac{1}{2} u_{\mathrm{e}} i_{\mathrm{eH} \beta} \\
& +\frac{1}{4} \hat{u}_{\mathrm{a}} \hat{i}_{\mathrm{a}} \cdot \sin \left(2 \cdot \gamma_{\mathrm{a}}-\varphi_{\mathrm{a}}\right) \\
& -\frac{1}{2} u_{\mathrm{a} 0} \hat{i}_{\mathrm{a}} \cdot \sin \left(\gamma_{\mathrm{a}}-\varphi_{\mathrm{a}}\right) \\
& +\frac{1}{2} u_{\mathrm{e}} \hat{i}_{\mathrm{eVp}} \cdot \sin \left(\gamma_{\mathrm{e}}\right) \\
& +\frac{1}{2} u_{\mathrm{e}} \hat{i}_{\mathrm{eVn}} \cdot \sin \left(-\gamma_{\mathrm{e}}+\varphi_{\mathrm{e}}\right) \\
p_{\Delta \alpha}= & +\frac{1}{2} u_{\mathrm{e}} \hat{i}_{\mathrm{a}} \cdot \cos \left(\gamma_{\mathrm{a}}-\varphi_{\mathrm{a}}\right) \\
+ & \hat{u}_{\mathrm{a}} \hat{i}_{\mathrm{eVn}} \cdot \sin \left(\gamma_{\mathrm{e}}-\varphi_{\mathrm{e}}\right) \cdot \sin \left(\gamma_{\mathrm{a}}\right) \\
p_{\Sigma 0}= & +\frac{1}{2} u_{\mathrm{e}} i_{\mathrm{eH} 0} \\
& -\frac{1}{4} \hat{u}_{\mathrm{a}} \hat{i}_{\mathrm{a}} \cdot \cos \left(\varphi_{\mathrm{a}}\right) \\
&
\end{aligned}
$$

$$
\begin{aligned}
& -\hat{u}_{\mathrm{a}} \hat{i}_{\mathrm{eVn}} \cdot \cos \left(\gamma_{\mathrm{e}}-\varphi_{\mathrm{e}}\right) \cdot \cos \left(\gamma_{\mathrm{a}}\right) \\
& -2 \hat{u}_{\mathrm{a}} i_{\mathrm{e} 0} \cdot \cos \left(\gamma_{\mathrm{a}}\right) \\
& -2 u_{\mathrm{a} 0} i_{\mathrm{e} \alpha} \\
& +\hat{u}_{\mathrm{a}} i_{\mathrm{eH} \beta} \cdot \sin \left(\gamma_{\mathrm{a}}\right) \\
& -\hat{u}_{\mathrm{a}} i_{\mathrm{eH} \alpha} \cdot \cos \left(\gamma_{\mathrm{a}}\right) \\
& +\hat{u}_{\mathrm{a}} \hat{i}_{\mathrm{eVp}} \cdot \sin \left(\gamma_{\mathrm{e}}\right) \cdot \sin \left(\gamma_{\mathrm{a}}\right) \\
& -\hat{u}_{\mathrm{a}} \hat{i}_{\mathrm{eVp}} \cdot \cos \left(\gamma_{\mathrm{e}}\right) \cdot \cos \left(\gamma_{\mathrm{a}}\right)
\end{aligned}
$$

$$
\begin{aligned}
p_{\Delta \beta}= & +\frac{1}{2} u_{\mathrm{e}} \hat{i}_{\mathrm{a}} \cdot \sin \left(\gamma_{\mathrm{a}}-\varphi_{\mathrm{a}}\right) \\
& +\hat{u}_{\mathrm{a}} \hat{i}_{\mathrm{eVn}} \cdot \sin \left(\gamma_{\mathrm{e}}-\varphi_{\mathrm{e}}\right) \cdot \cos \left(\gamma_{\mathrm{a}}\right) \\
& +\hat{u}_{\mathrm{a}} \hat{i}_{\mathrm{eVn}} \cdot \cos \left(\gamma_{\mathrm{e}}-\varphi_{\mathrm{e}}\right) \cdot \sin \left(\gamma_{\mathrm{a}}\right) \\
& -2 \hat{u}_{\mathrm{a}} i_{\mathrm{e} 0} \cdot \sin \left(\gamma_{\mathrm{a}}\right) \\
& -2 u_{\mathrm{a} 0} i_{\mathrm{e} \beta} \\
& +\hat{u}_{\mathrm{a}} i_{\mathrm{eH} \beta} \cdot \cos \left(\gamma_{\mathrm{a}}\right) \\
& +\hat{u}_{\mathrm{a}} i_{\mathrm{eH} \alpha} \cdot \sin \left(\gamma_{\mathrm{a}}\right) \\
& +\hat{u}_{\mathrm{a}} \hat{i}_{\mathrm{eVp}} \cdot \sin \left(\gamma_{\mathrm{e}}\right) \cdot \cos \left(\gamma_{\mathrm{a}}\right) \\
& +\hat{u}_{\mathrm{a}} \hat{i}_{\mathrm{eVp}} \cdot \cos \left(\gamma_{\mathrm{e}}\right) \cdot \sin \left(\gamma_{\mathrm{a}}\right)
\end{aligned}
$$

$$
\begin{aligned}
p_{\Delta 0}= & -\hat{u}_{\mathrm{a}} \hat{i}_{\mathrm{eVp}} \cdot \cos \left(\gamma_{\mathrm{e}}\right) \cdot \cos \left(\gamma_{\mathrm{a}}\right) \\
& -\hat{u}_{\mathrm{a}} \hat{i}_{\mathrm{eVp}} \cdot \sin \left(\gamma_{\mathrm{e}}\right) \cdot \sin \left(\gamma_{\mathrm{a}}\right) \\
& -2 u_{\mathrm{a} 0} i_{\mathrm{e} 0} \\
& -\hat{u}_{\mathrm{a}} i_{\mathrm{eH} \beta} \cdot \cos \left(\gamma_{\mathrm{a}}\right)
\end{aligned}
$$




$$
\begin{aligned}
& -\hat{u}_{\mathrm{a}} i_{\mathrm{eH} \alpha} \cdot \sin \left(\gamma_{\mathrm{a}}\right) \\
& -\hat{u}_{\mathrm{a}} \hat{i}_{\mathrm{eVn}} \cdot \cos \left(\gamma_{\mathrm{e}}-\varphi_{\mathrm{e}}\right) \cdot \cos \left(\gamma_{\mathrm{a}}\right) \\
& -\hat{u}_{\mathrm{a}} \hat{i}_{\mathrm{eVn}} \cdot \sin \left(\gamma_{\mathrm{e}}-\varphi_{\mathrm{e}}\right) \cdot \sin \left(\gamma_{\mathrm{a}}\right)
\end{aligned}
$$

$\hat{u}_{\mathrm{a}}$ represents the amplitude of the output voltage, $\hat{i}_{\mathrm{a}}$ the amplitude of the output current, $\gamma_{\mathrm{a}}$ the angle of the output voltage space vector and $\varphi_{\mathrm{a}}$ the phase shift between output voltage and output current. $u_{\mathrm{a} 0}$ is the zero sequence component of the output voltage and $u_{\mathrm{e}}$ is the input voltage.

\section{ACKNOWLEDGMENT}

The authors would like to thank the DFG (German Research Foundation) which finances this research project under grant number BR 1780/11-1.

\section{REFERENCES}

[1] J. Kolb, F. Kammerer, A. Schmitt, M. Gommeringer, and M. Braun, "The modular multilevel converter as universal high-precision 3ac voltage source for power hardware-in-the-loop systems," in PCIM Europe 2014; International Exhibition and Conference for Power Electronics, Intelligent Motion, Renewable Energy and Energy Management; Proceedings of, May 2014, pp. 1-8.

[2] J. Solanki, N. Frohleke, J. Bocker, and P. Wallmeier, "A modular multilevel converter based high-power high-current power supply," in Industrial Technology (ICIT), 2013 IEEE International Conference on, Feb 2013, pp. 444-450.

[3] T. Nakanishi and J.-I. Itoh, "Evaluation of isolated three-phase ac$\mathrm{dc}$ converter using modular multilevel converter topology," in Power Electronics Conference (IPEC-Hiroshima 2014 - ECCE-ASIA), 2014 International, May 2014, pp. 1095-1102.

[4] R. Marquardt, A. Lesnicar, and J. Hildinger, "Modulares Stromrichterkonzept für Netzkupplungsanwendungen bei hohen Spannungen," in ETG-Fachtagung, Bad Nauheim, Germany, 2002.

[5] A. Schmitt, M. Gommeringer, J. Kolb, and M. Braun, "A high current, high frequency modular multiphase multilevel converter for power hardware-in-the-loop emulation," in PCIM Europe 2014; International Exhibition and Conference for Power Electronics, Intelligent Motion, Renewable Energy and Energy Management; Proceedings of, May 2014, pp. 1-8.

[6] M. Manyage and P. Pillay, "New epstein frame for core loss measurements at high frequencies and high flux densities," in Industry Applications Society Annual Meeting, 2008. IAS '08. IEEE, Oct 2008, pp. 1-6.

[7] E. Nicol Hildebrand and H. Roehrdanz, "Losses in three-phase induction machines fed by pwm converter," Energy Conversion, IEEE Transactions on, vol. 16, no. 3, pp. 228-233, Sep 2001.

[8] J. Kolb, F. Kammerer, M. Gommeringer, and M. Braun, "Cascaded control system of the modular multilevel converter for feeding variablespeed drives," Power Electronics, IEEE Transactions on, vol. 30, no. 1, pp. 349-357, Jan 2015.

[9] D. Schröeder, Elektrische Antriebe - Regelung von Antriebssystemen. Springer Berlin Heidelberg, 2009.

[10] C. Ackermann, "Optimale Regelung der permanentmangeterregten Synchronmaschine unter Nutzung des Reluktanzmoments," Ph.D. dissertation, Karlsruher Institut für Technologie, Elektrotechnisches Institut, 2012. 